\title{
Chemical bile duct embolization for chemical hepatectomy, long term efficacy and feasibility in rats
}

\author{
Anuj Shrestha1,2, Yong Zhou', Hui Mao³, Fu-yu Li¹, Wenjie Ma1, Qin Yang', Yong-qiong Zhang', Huan Feng', \\ Wei Zhang ${ }^{4}$, Nan-sheng Cheng ${ }^{1}$ \\ ${ }^{1}$ Department of Hepatobiliary Surgery, West China Hospital of Sichuan University, Chengdu, China, ${ }^{2}$ Department of General Surgery, Gandaki \\ Medical College, Pokhara, Nepal, ${ }^{3}$ Department of Medicine, West China Hospital of Sichuan University, Chengdu, China, ${ }^{4}$ Department of \\ Medicine, The George Washington University, USA
}

\section{A B S TR A C T}

Background: Hepatolithiasis is the presence of calculi within the intrahepatic bile ducts. It represents a significant problem in hepatobiliary surgery because of its high recurrence rate and the associated intra-operative and post-operative risks. This study was designed to explore the long-term efficacy of chemical biliary duct embolization (CBDE) to treat recurrent hepatolithiasis and to explain the mechanism of CBDE. Aims and Objectives: To investigate the long term efficacy of chemical bile duct embolization (CBDE) on the targeted hepatic lobe and to explain the mechanism of CBDE to achieve chemical hepatectomy. Materials and Methods: The median biliary duct of rats were embolized with phenol and N-butylcyanoacrylate. The short-term (6 weeks) and long-term (12 weeks) effects of chemical bile duct embolization were compared by observing the degree of atrophy, fibrosis, and proliferation of collagen fibers and apoptosis of hepatocytes of the embolized hepatic lobe. The feasibility and effectiveness of chemical hepatectomy were analyzed by histology, Western blot analysis of collagen I fibers and assessment of hydroxyproline content. Results: After 6 weeks of the procedure, destruction of hepatocytes, fibrosis and "self-cut" was seen only in the periphery of the targeted hepatic lobe. Whereas after 12 weeks, complete destruction of hepatocytes, and replacement with proliferative bile ductules and collagen fibers leading to complete fibrosis and "self-cut" phenomenon in the whole targeted hepatic lobe was seen. Collagen I expression in the 6-weeks treatment group and 12-weeks treatment groups were 4 times and 12 times higher than that in the Sham operated (SO) group respectively $(\mathrm{P}<0.05)$. In addition, there was increase in hepatic hydroxyproline content (HYP) approximately by sevenfold in 6-weeks treatment group and twentyfold in 12-weeks treatment group after CBDE, when compared to that in the SO group $(P<0.05)$. Conclusion: Chemical bile duct embolization can achieve ideal effect of chemical hepatectomy in the whole targeted lobe.

Access this article online

Website:

http://nepjol.info/index.php/AJMS DOI: 10.3126/ajms.v7i3.13635 E-ISSN: 2091-0576

P-ISSN: 2467-9100

Key words: Hepatolithiasis, Bile duct, Chemical embolization, Chemical hepatectomy

\section{INTRODUCTION}

Hepatolithiasis is commonly encountered in the AsiaPacific regions, but is considered as a particularly intractable disease because of its recalcitrant nature. ${ }^{1,2}$ Preventing stone recurrence and biliary re-stenosis after successful removal of intrahepatic stones still remains an unachieved goal in hepatobiliary surgery., ${ }^{3,4}$ Patients who requires third or fourth biliary surgery for the stone recurrence along with severe biliary stricture, are commonly encountered in East Asia. In fact, approximately two-thirds of patients with hepatolithiasis in Asia require follow-up surgery due to recurrent biliary stricture, bile duct infection, and other refractory factors. The degree of biliary stricture is directly proportional to the frequency of stone recurrence. ${ }^{2}$ This is further associated with gradual worsening of the 
patient's overall health, and thus increasing intra-operative risk. Currently, there is no effective method for prevention of intrahepatic bile duct stenosis and post-operative recurrences of biliary calculi available. ${ }^{5}$

The ultimate goal of hepatolithiasis treatment is clearance of the stone, correction of biliary stricture, and removal of diseased liver segments. Conventional hepatectomy for treatment of stones and biliary duct stenosis localized in one segment can achieve a good result in about $91.60 \%$ of the patient. But, about $16.0 \%$ of the patients undergoing hepatectomy may have recurrence of stone at other site in the liver. ${ }^{6,7}$ In addition, whole-liver distribution of stones can be seen in $40 \%$ of the hepatolithiasis patients, and conventional hepatectomy, especially resection of the right posterior or caudate lobe, carries a great operative risk and trauma. Moreover, it is unfeasible to repeatedly perform a hepatic lobe/segment resection because of the dense perihepatic adhesions due to repeated infections, repeated operations, and past liver abscesses that ruptured into the perihepatic space. Due to these reasons, application of hepatectomy is highly restricted. The use of choledochoscopy has greatly increased stone clearance rates. However, stone recurrence is inevitable if biliary stricture is not corrected, thus resulting multiple operations or choledocholithotomy. Therefore, new therapeutic approach for the treatment and prevention of hepatolithiasis and its recurrence is urgently required., 3

It is a well-known fact that hepatolithiasis is a segmental disease, characterized by its strict distribution within the intrahepatic biliary system, and biliary obstruction can induce hepatic atrophy, fibrosis, and "self-cut" chemical hepatectomy in its subsidiary hepatic segment. ${ }^{5,9}$ In previous study, we proved the practicability of chemical bile duct embolization (CBDE) to induce hepatic atrophy and chemical hepatectomy of the targeted hepatic lobe. However, hepatic atrophy, fibrosis, and "self-cut" phenomenon was only achieved in the peripheral region of the targeted hepatic lobe. ${ }^{5,10-15}$ It is still unknown whether hepatic atrophy, fibrosis and "self-cut" phenomenon can be achieved in the whole embolized lobe by CBDE in a longer period of time. Thus, this study was designed to differentiate and explore the efficacy and feasibility of the short-term (6 weeks) and long-term (12 weeks) effects of CBDE to achieve the desired effect of chemical hepatectomy ("self-cut") in the whole targeted hepatic lobe.

\section{MATERIALS AND METHODS}

\section{Experimental program}

All animal experiments were approved by the Animal Care and Use Committee of Sichuan University (Chengdu,
China). Twenty-six male Sprague-Dawley rats, weighing 220-250 gm provided by Experimental Animal Centre of Sichuan University (Chengdu, China), were randomly divided into three groups depending on their treatment group and time of tissue collection:

(a) Tissue collected 6 weeks after embolization (6-weeks treatment group, $\mathrm{n}=10)$ : The median biliary duct was ligated and was injected with $0.15 \mathrm{ml}$ of phenol through a $\mathrm{T}_{26} \mathrm{G}$ polyethylene catheter (Abbott, Co., Chicago, IL, USA), and aspirated after 15 minutes. Subsequently, $0.15 \mathrm{ml}$ of $\mathrm{N}$-butyl-cyanonacrylate (Tissue adhesive glue, Jingya Co., Beijing, China) was injected quickly with pressure. Rats were sacrificed at the sixth week. Embolized lobe and non-embolized lobe were resected out and preserved for further analysis.

(b) Tissue collected 12 weeks after embolization (12-weeks treatment group, $n=10)$ : The median biliary duct was embolized as above. After retrograde cholangiography, rats were sacrificed at the twelfth week after the model establishment. Embolized lobe and non-embolized lobe were resected out and preserved for further analysis.

(c) Sham operation (SO group, $\mathrm{n}=6$ ): The medial biliary duct was dissected out only.

\section{Morphological and histological analyses}

After all rats were sacrificed on $6^{\text {th }}$ week and $12^{\text {th }}$ week respectively, the liver samples were taken and fixed in liquid nitrogen and 10\% formaldehyde for further tests. Five micrometer thick formalin-fixed liver tissue slides were prepared for HE staining. The percentage of the fibrosis area was evaluated by Masson staining.

\section{Western blot analysis of collagen I}

Hundred micrograms of extracted protein were separated by sodium dodecyl sulfate polyacrylamide gel electrophoresis, and then electrophoretically transferred to polyvinylidinedifluoride membrane and hybridized. The membrane was blocked with 5\% non-fat dry milk for $1 \mathrm{~h}$ then incubated with collagen I primary antibody (Zymed Co., South San Francisco, California, USA) overnight at $4^{\circ} \mathrm{C}$. After rinsing with Tris-buffered saline and Tween 20, the membrane was incubated for $2 \mathrm{~h}$ with horseradish peroxidase-conjugated secondary antibody. Immunoreactive bands were visualized with enhanced chemi-luminescence and captured on X-ray film. The results were expressed as the ratio of the expression of target gene to that of $\beta$-actin.

\section{Assessment of hydroxyproline content ( $\mathrm{mg} / \mathrm{g}$ liver)} Collagen content was quantitatively determined by hydroxyproline, an amino acid found primarily in collagen and the principal component of the extracellular matrix. 
The hydroxyproline content in liver was measured as per the instruction provided byJamall et al. ${ }^{16,17}$

\section{Statistical analysis}

Data analyses were made using SPSS 10.0 software (SPSS Inc., Chicago, and IL., USA). Quantitative data were expressed as mean and standard deviation. Statistical comparisons among means were performed using one-way analysis of variance (ANOVA), and Fisher's Least Significant Difference analysis was used for within-group comparisons. $P<0.05$ was considered statistically significant.

\section{RESULTS}

\section{Histopathological examination}

In the 6-weeks treatment group, the hepatocytes in the peripheral region were almost completely destroyed and replaced with proliferative bile ductules and collagen fibers leaving large islands of residual liver cells in the center portion of the targeted hepatic lobe (Figure 1a). Whereas, in 12-weeks treatment group, the hepatocytes disappeared completely and were replaced by proliferative ductles and collagen fibers leading to complete fibrosis and "selfcut" phenomenon in almost the whole embolized lobe (Figure 1b). Fibrosis levels in both treatment groups were expressed using Masson staining. Blue stained collagen fibers portions were significantly higher in 12 -weeks treatment group (Figure 1d) in comparision to that of 6-weeks treatment group (Figure 1c). No liver abscess and alteration in the liver functions were seen during the entire experiment.

\section{Western blot analysis of collagen I}

Collagen I protein expression in the 6-weeks treatment group was 4 times higher than that in the SO group. Whereas, collagen I expression in the 12-weeks treatment

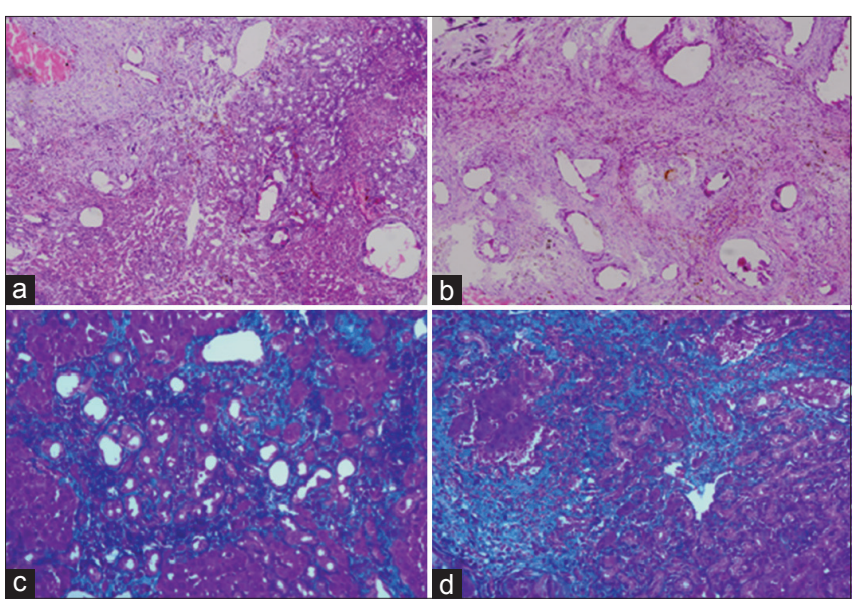

Figure 1: HE and Masson staining of embolized lobe: effects after 6 weeks on the targeted lobe $(a, c)$; effects after 12 weeks on the targeted lobe $(b, d)$. group was 12 times higher than that in the $\mathrm{SO}$ group, which was 3 times higher than that in the 6-weeks treatment group (Figure 2) $(P<0.05)$.

\section{Assessment of hepatic hydroxyproline content}

Quantitative determination of hepatic collagen content was performed by assessing the hepatic hydroxyproline (HYP) content after CBDE. There was an approximate sevenfold increase in 6-weeks treatment group and a twentyfold increase in 12-weeks treatment group, when compared to that in the SO group. Additionally, the differences between the 6-weeks treatment group and 12 -weeks treatment group were statistically significant $(P<0.05)$ (Figure 3 ).

\section{DISCUSSION}

Hepatolithiasis is a commonly encountered disease in the Asia-Pacific region, but is still regarded intractable as the long term effects of current available therapeutic approaches are far from satisfactory. ${ }^{1,18}$ Calculi in secondary or tertiary subsidiary branches of bile duct are usually complicated with biliary duct stenosis. Therefore, even after successful stone clearance with choledochoscopy, stone recurrence is inevitable. Biliary stricture causes bile stasis and secondary infections which then stimulates hyperplasia of bile duct thus causing chronic proliferative cholangitis (CPC). Repeated episodes of CPC leads to stone formation, thus forming a vicious cycle of $\mathrm{CPC}$ and stone formation. ${ }^{2,19}$ Therefore, treatment of hepatolithiasis must include clearance of stones, correction of biliary stricture and elimination of CPC. Presently, traditional hepatectomy remains the only treatment of choice for hepatolithiasis. Unfortunately, $40 \%$ of hepatolithiasis patients have calculi distributed throughout the liver and most patients cannot tolerate multiple lobes/segments resection. Due to these reasons, application of hepatectomy to treat hepatolithiasis is greatly restricted. In previous study, we explored the feasibility and efficacy of filling the diseased bile duct with chemical embolization agent to achieve chemical resection of the diseased bile duct and hepatic segment, and was able to establish the effects of chemical hepatectomy only in the periphery of the targeted hepatic lobe. ${ }^{11-15}$ This study focuses on whether $\mathrm{CBDE}$ in the long-term manages to achieve complete fibrosis, "self-cut" and chemical hepatectomy in the whole embolized hepatic lobe.

In both the 6-weeks and 12 weeks treatment groups, chemical hepatectomy resulted from CBDE exhibited destruction of the biliary duct mucosa and replacement with collagen fibers of the targeted hepatic lobe. Thus, completely eradicating the pathological basis of stone recurrence: chronic proliferative cholangitis (СРC). In the 6-week treatment group, islands of residual hepatocytes were still visible, with signs of 


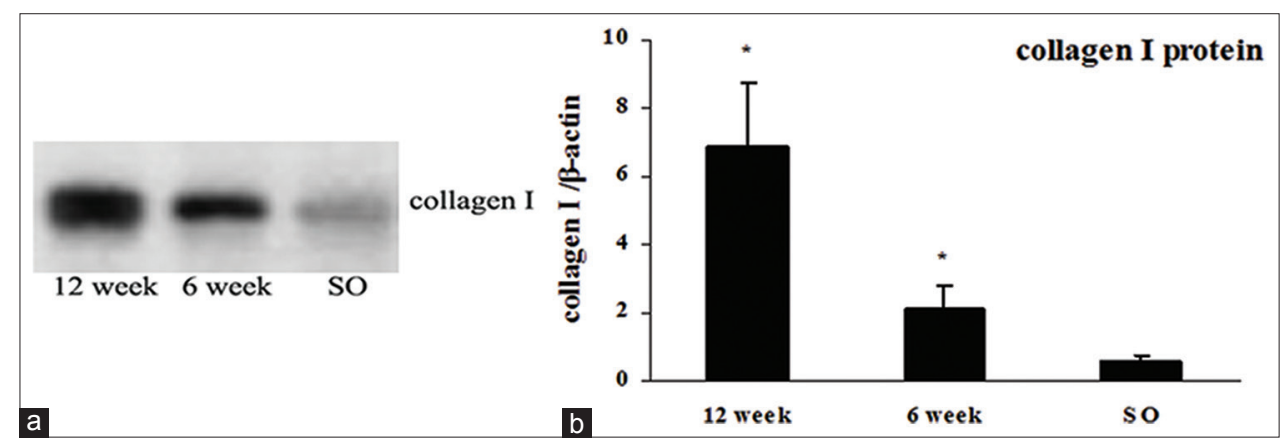

Figure 2: Western blot analysis of Collagen I. Collagen I expression was significantly higher in the embolized lobes. Collagen I protein expression in the 6-week treatment group was found to be 4 times higher than that in the SO group. Whereas, collagen I expression in the 12-week treatment group was 12 times higher than that in the SO group

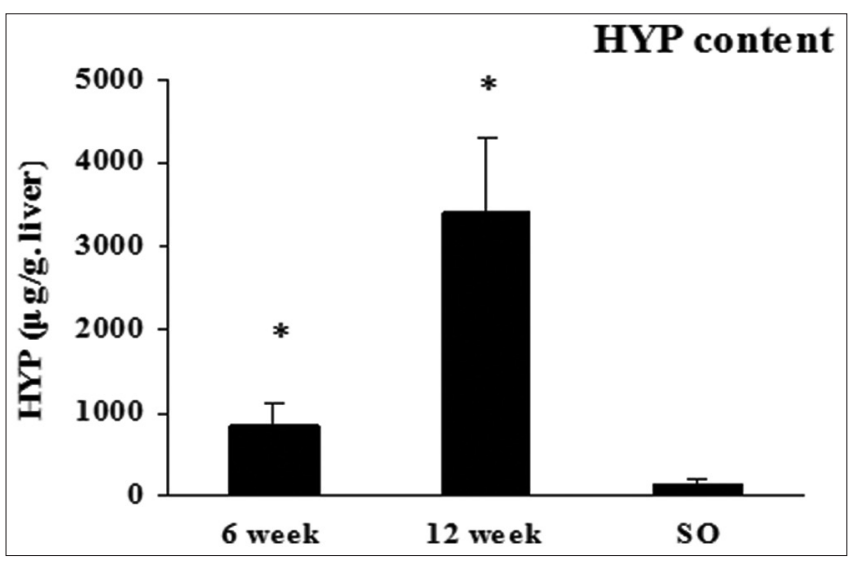

Figure 3: Comparison of the changes of hydroxyproline content following CBDE treatment. HYP content in the 6-week treatment group was found to be 7 times higher than that in the SO group. Whereas, HYP content in the 12-week treatment group was 20 times higher than that in the $\mathrm{SO}$ group. The difference between the 6-weeks treatment group and 12-weeks treatment group was statistically significant $(P<0.05)$.

chemical hepatectomy only observed in the periphery of the embolized lobe. Whereas in the 12-week treatment group, destruction of hepatocytes, fibrosis and "self-cut" was seen in the whole embolized hepatic lobe. Masson staining revealed collagen fibers were seen in almost the entire embolized liver lobe. This was confirmed quantitatively by western blot analysis of the collagen fiber. The expression level of collagen I at 6 and 12 weeks after CBDE was 4 and 12 times higher than the corresponding levels in the SO group respectively. In addition, the hydroxyproline content in 6-weeks treatment group and 12-weeks treatment group were 7 -times and 20-times higher than that in the SO group respectively. The difference of hydroxyproline content in the 6-weeks and 12-weeks treatment group were also statistically significant. Therefore, CBDE can achieve complete chemical hepatectomy of the embolized lobe. No liver abscess was seen during the entire experiment. The bile duct, portal vein and hepatic artery are enclosed in a dense and fibrous Glisson sheath. After embolization of the bile duct, it obviously distends and directly compresses the adjacent blood vessels. As this obstruction is a slow and gradual process, establishment of secondary collateral circulation to the targeted hepatic lobe is possible. Reservation of hepatic artery, secondary establishment of collateral circulation, selection of smaller subsidiary bile ducts and appropriate embolization agent all help to prevent the occurrence of liver abscess. ${ }^{20,21}$

Feasibility of embolization of small bile ducts to treat hepatolithiasis have been confirmed in the past. Patients with multiple biliary calculi with biliary stricture in the inferior branch of the left lateral bile duct were treated with CBDE. Fifteen months later, computed tomography scans demonstrated that the inferior segment of the left lateral lobe had almost completely atrophied and disappeared, with no signs of either hepatic abscess or stone recurrence. ${ }^{22}$ Liver abscess is rarely encountered in totally atrophic lobe. Liver abscess may occur secondary to stone obstruction or incomplete embolization of the first or second subsidiary bile duct. If the bigger bile duct is targeted, occurrence of liver abscesses is likely due to incomplete embolization of the targeted bile duct. Selection of an appropriate embolization agent that can complete embolization hepatic ducts of various sizes is the most important factor for successful chemical hepatectomy via CBDE. 5,12 Fu-Yu li, Jing-Qiu Cheng et al ${ }^{12}$ suggested that phenol is not only the most efficient sclerosants of gall bladder mucosa, but is also a powerful antiseptic which might not only assist in ameliorating biliary infection which commonly accompanies hepatolithiasis, but also could commendably destroy the biliary mucosa and induced fibrous conglutination in the bile duct lumen, leading to partial occlusion of the large-size and middle-size biliary ducts, and complete occlusion of the small biliary ducts. ${ }^{18,22,23}$ However, the biliary system could not be permanently occluded by using phenol or ethanol alone due to the regeneration of biliary mucosa. ${ }^{22}$ As an ideal liquid occlusion agent; cyanoacrylate, histoacryl or neoprene has such remarkable liquid-dynamic characteristics that could perfectly fill bile ducts of various sizes, including the acroteric branch. Thus, leading to complete disappearance 
of mucosal epithelia which are entirely replaced by foreign body granuloma and collagen fibers. Additionally, cyanoacrylate occupies the space where stones can occur, thus preventing intrahepatic stone recurrences. All of these suggests that mucosal sclerosant plus tissue adhesive glue results in a more complete and reliable embolization than a mucosal sclerosant alone. In addition, histoacryl (also called N-butyl cyanoacrylate), a derivative of cyanoacrylate can be dissolved in contrast medium and thus can be visualized under $\mathrm{x}$-ray. This kind of visibility makes CBDE much safer, easier and more accurate in its application. ${ }^{5,11,12,15,23-25}$

The mechanism underlying chemical hepatectomy via CBDE may involve the following aspects. The embolized bile duct obviously distends and compresses the adjacent blood vessels, thus reducing the blood supply to the embolized hepatic lobe. ${ }^{15,26-28}$ Reduction in blood supply to the embolized lobe and deposition of large amount of intrahepatic bile salts due to biliary obstruction activates hepatocellular apoptosis via upregulation of Fas and Bax, which then activates capsase- 3 and capsase-9. Fas- triggered apoptosis activates hepatic stellate cells (HSC), which secretes TGF- $\beta_{1}$ and TIMP ${ }_{1}$ and consequently synthesize large amount of collagen fibers. Additionally, TGF- $\beta_{1}$ and TIMP $_{1}$ can accelerate hepatocellular apoptosis and inhibit hepatocellular regeneration. Thus, a perpetual cycle of hepatocytes death and proliferation of bile ductules and collagen may develop. It is important to note that CBDE not only permanently embolize, destroy the biliary tree and reduce the blood supply of target liver segment, but also compresses the microvasculature of hepatocytes and replaces hepatocytes by large number of proliferated intrahepatic bile ductules and collagen fibers. With the acceleration of hepatic fibrosis, the blood supply of embolized lobe reduces. Liver cells can thus be induced ongoing injury and apoptosis. Therefore, hepatic fibrosis process caused by CBDE may progress continuously until complete hepatic fibrosis and "self-cut" chemical hepatectomy is achieved. 12,27,29-31

CBDE for chemical hepatectomy may provide an innovative and less invasive therapeutic approach in treating hepatolithiasis and preventing postoperative recurrences of intrahepatic stones. Chemical hepatectomy of the targeted liver segment is more selective than the traditional hepatectomy, as it can be implicated accurately for subsegmental hepatectomy and preserve significant functional hepatocytes. However, just like chemical cholecystectomy, CBDE is feasible but not recommended routinely. We must emphasize that cholangioscopic lithotomy or open hepatectomy are still the best treatments of choice for routine hepatolithiasis. ${ }^{7,23,28}$ However, most of the latestage hepatolithiasis patients cannot tolerate multiple hepatic lobe or segment resections, since intrahepatic calculi can cause many pathological lesions to the liver such as secondary biliary cirrhosis, multiple calculi, recurrent cholangitis, repeated operation, etc. In addition, recurrent hepatolithiasis with cholangitis in these latestage hepatolithiasis patient can lead to development of apical epithelial hyperplasia, which may progress to cholangiocarcinoma. Thus, early eradication of this kind of chronic proliferative inflammation can prevent occurrence of concomitant intrahepatic cholangiocarcinoma. Therefore, application of CBDE in these conditions not only avoids trauma and risks of conventional hepatectomy, but also expands the operative indications for those latestage hepatolithiasis patients who are at a high risk for hepatectomy. ${ }^{5}$ Furthermore, if patients with multiple calculi combined with a biliary stricture are only treated by endoscopic cholelithotomy, recurrence of the intrahepatic stone is foreseeable. Thus, application of CBDE after choledochoscopic lithotomy would certainly reduce re-operations for recurrent hepatolithiasis. However, the targeted biliary duct in these patients should be limited to the third subsidiary bile ducts, which are easy to occlude completely without subsequent development of cholangitis or liver abscess. If the first or the second subsidiary bile duct is chosen as the target biliary duct, one has to be more careful, as there is a greater risk of suppurative cholangitis or liver abscess due to incomplete embolization..$^{3-5,8}$

In our study, we were able to conclude that CBDE after 12 weeks was able to express fibrosis, proliferation of bile ductules and "self-cut" of the whole embolized lobe thus achieving the ideal effect of chemical hepatectomy. We believe CBDE might be able to partially replace conventional hepatectomy as a more feasible, effective and safe approach to treat recurrent hepatolithiasis. However, relative literatures suggesting the result are very few and are still in its preliminary stage. Thus, further research and analysis about its clinical significance, indications, contraindications, precautionary measures and long-term effectiveness is definitely required prior to its clinical application.

\section{ACKNOWLEDGEMENT}

\section{Funding}

We acknowledge the financial support from the National Nature Science Foundation of China (30801111, 30972923) and Science \& Technology Support Project of Sichuan Province (14ZC1337 and 14ZC1335).

\section{Conflict of interest}

All authors declare that they have no conflict of interest.

\section{Ethical approval}

All animal experiments were approved by the Animal Care and Use Committee of Sichuan University (Chengdu, China). 


\section{REFERENCES}

1. Chen XG, Liu JQ, Peng MH, Wang WG, Yang DH, Hu DH, et al Clinical epidemiological study on intrahepatic cholelithiasis: analysis of 8585 cases. Hepatobiliary Pancreat Dis Int 2003;2(2):281-284.

2. Li FY, Cheng NS, Mao H, Jiang LS, Cheng JQ, Li QS, et al. Significance of Controlling Chronic Proliferative Cholangitis in the Treatment of Hepatolithiasis. World Journal of Surgery 2009;33(10):2155-2160.

3. Lee SK, Seo DW, Myung SJ, Park ET, Lim BC, Kim HJ, et al. Percutaneous transhepatic cholangioscopic treatment for hepatolithiasis: an evaluation of long-term results and risk factors for recurrence. Gastrointest Endosc 2001;53(3):318-323.

4. Hwang JH, Yoon YB, Kim YT, Cheon JH and Jeong JB. Risk factors for recurrent cholangitis after initial hepatolithiasis treatment. J Clin Gastroenterol 2004;38(4):364-367.

5. Li FY, Jiang LS, de Jong MC, Cheng NS, Cheng JQ, Li N, et al. Clinical prospect of applying the chemical bile duct embolization to achieve a chemical hepatectomy in the treatment of highly selected hepatolithiasis. Surg Laparosc Endosc Percutan Tech 2009;19(3):183-187; discussion 7-9.

6. Uchiyama $\mathrm{K}$, Onishi $\mathrm{H}$, Tani $\mathrm{M}$, Kinoshita $\mathrm{H}$, Ueno $\mathrm{M}$ and Yamaue $\mathrm{H}$. Indication and procedure for treatment of hepatolithiasis. Arch Surg 2002;137(2):149-153.

7. Lee TY, Chen YL, Chang HC, Chan CP and Kuo SJ. Outcomes of hepatectomy for hepatolithiasis. World Journal of Surgery 2007;31(3):479-482.

8. He XD, Liu W, Li BL, Zhang ZH and Zhang JX. Combined surgical therapy for hepatolithiasis. Chinese medical sciences journal $=$ Chung-kuo i hsueh k'o hsueh tsa chih/Chinese Academy of Medical Sciences 2005;20(2):123-125.

9. Huang Z. Development of biliary duct surgery. Biliary duct surgery of huang zhi qiang. Jinan: Shandong Science and Technology press 2000.

10. Li FY, Li N, Jiang LS, Cheng JQ, Cheng NS, Wu XW, et al. Feasibility and effectiveness of chemical bile duct embolization for chemical hepatectomy: a preliminary study. Hepatobiliary Pancreat Dis Int 2006;5(4):570-573.

11. Li FY, Cheng JQ, Mao H, Li N, Jiang LS, He S, et al. Preliminary study of chemical bile duct embolization to treat hepatolithiasis in rabbits. J Gastroenterol Hepatol 2006;21(6):994-998.

12. Li FY, Cheng JQ, Li N, He S, Zhang MM, Dong JH, et al. Effectiveness of chemical biliary duct embolization for chemical hepatectomy. J Gastroenterol Hepatol 2006;21(5):880-886.

13. Li FY, Cheng JQ, Mao H, Li N, Jiang LS, Cheng NS, et al. Treatment of hepatolithiasis by endoscopic chemoembolization of the left hepatic duct. Endoscopy 2006;38(8):845-847.

14. Li FY, Cheng JQ, He S, Li N, Zhang MM, Dong JH, et al. A preliminary study of applying chemical biliary duct embolization to chemical hepatectomy in rats. Dig Dis Sci 2005;50(6):1161-1165.

15. Li F, Cheng J, He S, Li N, Zhang M, Dong J, et al. The practical value of applying chemical biliary duct embolization to chemical hepatectomy for treatment of hepatolithiasis. Journal of Surgical Research 2005;127(2):131-138.

16. Jamall IS, Finelli VN and Que Hee SS. A simple method to determine nanogram levels of 4-hydroxyproline in biological tissues. Analytical biochemistry 1981;112(1):70-75.

17. Gerling B, Becker $M$, Waldschmidt J, Rehmann $M$ and Schuppan D. Elevated serum aminoterminal procollagen typeIII-peptide parallels collagen accumulation in rats with secondary biliary fibrosis. J Hepatol 1996;25(1):79-84.

18. Terao $R$, Honda $K$, Hatano $E$, Uehara $T$, Yamamoto $M$ and Yamaoka Y. Suppression of proliferative cholangitis in a rat model with direct adenovirus-mediated retinoblastoma gene transfer to the biliary tract. Hepatology 1998;28(3):605-612.

19. Li F, Zhou Y, Cheng N, Mao H, Jiang L, Li N, et al. Epidermal growth factor receptor as a target for anti-proliferative treatment of proliferative cholangitis in hepatolithiasis. Journal of Surgical Research 2011;166(1):87-94.

20. Kyokane T, Nagino M, Kamiya J, Nimura $Y$ and Nagasaka T. Simultaneous segmental obstruction of bile duct and portal vein markedly changes a population of biliary and hepatic cells in human liver. Langenbecks Archives of Surgery 2003;388(4):270-275.

21. Ersoz S, Cakmakh S, Gecim E, Turkcapar AG and Anadol E. The safety of total hepatic vascular occlusion in rabbits with acute extrahepatic cholestasis. Eur Surg Res 1996;28(2):104-110.

22. Leahy AL, McCollum PT, O'Gorman S, Darzi A, Marks P, Kay E, et al. Cystic duct obliteration and gallbladder mucosal destruction: a feasible alternative to cholecystectomy. Br J Surg 1991;78(11):1321-1324.

23. Xu Z, Wang L, Zhang N, Ling X, Hou C and Zhou X. Chemical ablation of the gallbladder: clinical application and long-term observations. Surg Endosc 2005;19(5):693-696.

24. Ohara S, Onoyama H, Tomita $M$ and Yamamoto $M$. Primary and experimental study of a new treatment for hepatolithiasis by injection of resin in the intrahepatic bile duct. Nippon Geka Gakkai Zasshi 1995;96(8):563-568.

25. Memis A, Oran I and Parildar M. Use of histoacryl and a covered nitinol stent to treat a bronchobiliary fistula. J Vasc Interv Radiol 2000;11(10):1337-1340.

26. Ni Y, Lukito G, Marchal G, Cresens E, Yu J, Petre C, et al. Potential role of bile duct collaterals in the recovery of the biliary obstruction: experimental study in rats using microcholangiography, histology, serology and magnetic resonance imaging. Hepatology 1994;20(6):1557-1566.

27. Nakano $\mathrm{S}$, Haratake $\mathrm{J}$ and Hashimoto $\mathrm{H}$. Alterations in bile ducts and peribiliary microcirculation in rats after common bile duct ligation. Hepatology 1995;21(5):1380-1386.

28. Kyokane T, Nagino M, Oda K and Nimura Y. An experimental study of selective intrahepatic biliary ablation with ethanol. Journal of Surgical Research 2001;96(2):188-196.

29. Canbay A, Higuchi H, Bronk SF, Taniai M, Sebo TJ and Gores GJ. Fas enhances fibrogenesis in the bile duct ligated mouse: a link between apoptosis and fibrosis. Gastroenterology 2002;123(4):1323-1330.

30. Takiya S, Tagaya T, Takahashi K, Kawashima H, Kamiya M, Fukuzawa $Y$, et al. Role of transforming growth factor beta 1 on hepatic regeneration and apoptosis in liver diseases. Journal of clinical pathology 1995;48(12):1093-1097.

31. Friedman SL. Molecular mechanisms of hepatic fibrosis and principles of therapy. J Gastroenterol 1997;32(3):424-430.

\section{Authors Contribution:}

AS and YZ - Contributed to the data acquisition and analysis and drafted the manuscript; WJM, HF and QY - Contributed in histology, western blot analysis and data acquisition; WZ, YQZ and CNS - Were involved in the revision of the manuscript; LFY and HM - Contributed to the study design and revision of the manuscript.

Foundation Item:

We acknowledge the financial support from the National Nature Science Foundation of China $(30801111,30972923)$ and Science \& Technology Support Project of Sichuan Province (14ZC1337 and 14ZC1335).

Source of Support: None decleared. Conflict of Interest: None. 